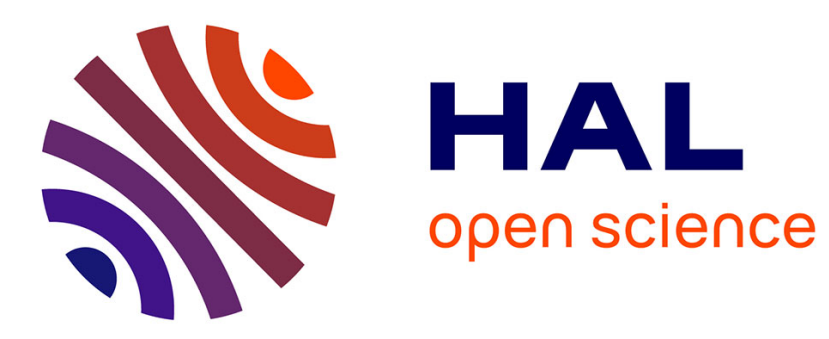

\title{
On the tearing of thin sheets
}

Elsa Bayart, A. Boudaoud, Mohktar Adda-Bedia

\section{To cite this version:}

Elsa Bayart, A. Boudaoud, Mohktar Adda-Bedia. On the tearing of thin sheets. Engineering Fracture Mechanics, 2010, 77 (11), pp.1849-1856. 10.1016/j.engfracmech.2010.03.006 . hal-03025036

\section{HAL Id: hal-03025036 https://hal.science/hal-03025036}

Submitted on 4 Jan 2021

HAL is a multi-disciplinary open access archive for the deposit and dissemination of scientific research documents, whether they are published or not. The documents may come from teaching and research institutions in France or abroad, or from public or private research centers.
L'archive ouverte pluridisciplinaire HAL, est destinée au dépôt et à la diffusion de documents scientifiques de niveau recherche, publiés ou non, émanant des établissements d'enseignement et de recherche français ou étrangers, des laboratoires publics ou privés. 


\title{
On the tearing of thin sheets
}

\author{
E. Bayart, A. Boudaoud and M. Adda-Bedia \\ Laboratoire de Physique Statistique, ENS, Paris VI, Paris VII, CNRS, \\ 24 rue Lhomond, 75005 Paris, France.
}

\begin{abstract}
We perform an experimental study to investigate the propagation of one or two cracks in a thin elastic sheet of a brittle material torn in an out-of-plane shear mode. We observe that a single crack always follows a straight path, whereas two cracks propagating simultaneously follow curved paths and merge, forming a tongue-like shape. The present experimental setup allows the understanding of how the energy introduced at a large scale is focused at the crack tip. We find that the geometry of the sheet is determined by the direction of a large scale force, applied to the crack tip, which is perpendicular to cracked surfaces. While the material is deformed at large scales under mode III loading, the geometry of system in the vicinity of the crack tip adapts such that the material is locally broken under a pure mode I loading.
\end{abstract}

Key words: A. Crack path; Thin plate theory; Crack stability; B. Crack mechanics.

\section{Introduction}

Crack path prediction is one of the main challenges in the field of fracture mechanics. Indeed, a satisfactory equation of motion of a crack tip requires a fundamental understanding of material separation mechanisms $(1 ; 2 ; 3 ; 4)$. Within the framework of Linear Elastic Fracture Mechanics (LEFM), the propagation of a crack is mainly governed by the singular behavior of the stress field in the vicinity of its tip $(1 ; 2)$. For a two-dimensional quasi-static crack submitted to in-plane loading (mode I+II), this behavior is given by

$$
\sigma_{i j}(r, \phi)=\frac{K_{\mathrm{I}}}{\sqrt{2 \pi r}} \Sigma_{i j}^{\mathrm{I}}(\phi)+\frac{K_{\mathrm{II}}}{\sqrt{2 \pi r}} \Sigma_{i j}^{\mathrm{II}}(\phi)+O\left(r^{0}\right),
$$

where $\Sigma_{i j}^{\mathrm{I}}(\phi)$ and $\Sigma_{i j}^{\mathrm{II}}(\phi)$ are universal functions describing the angular variation of the stress field, and $K_{\mathrm{I}}$ and $K_{\mathrm{II}}$ are the stress intensity factors (SIFs). 
The evolution of the crack tip is governed by the Griffith energy criterion $(1 ; 2 ; 5)$, which states that the intensity of the loading necessary to induce propagation is given by

$$
G=\Gamma
$$

where $G$ is the energy release rate and $\Gamma$ is the fracture energy of the material, that is the energy needed to create new free surfaces. While the Griffith criterion is very useful in predicting crack initiation, it cannot predict the direction of the crack tip, and therefore it is not sufficient to determine the actual path of the crack. In order to achieve this, several suggestions have been made. Among them, the Principle of Local Symmetry (PLS) states that the crack advances in such a way that in-plane shear stress vanishes in the vicinity of the crack tip, or explicitly

$$
K_{\mathrm{II}}=0,
$$

This rule was proposed for in-plane quasi-static cracks $(6 ; 7)$, and then generalized to rapidly moving cracks (4). A different approach, known as the maximum energy release rate criterion (8) states that the crack advances in a direction that maximizes its energy release rate. Interestingly, even though this approach is quite different from the PLS, it produces very similar results to those obtained using the PLS to such an extent that they were even conjectured to coincide (9). However, as shown in (10) for kinked cracks and in (11) for branched cracks, the results are not exactly the same and a clear distinction can be made between the two. In fact, it has been shown that the PLS is the only self-consistent criterion $(7 ; 2)$.

While the stability of crack paths under in-plane deformations has received extensive attention, see e.g. $(3 ; 12)$, there exists few experimental observations and theoretical predictions on the stability of crack propagation under out-ofplane shear deformations (mode III) in brittle materials $(13 ; 14)$. Note however that from a historical point of view, the first criterion for crack path selection, based on symmetry arguments similar to the PLS, was first formulated for mode III crack propagation (15). In the case of thin films, such a configuration is illustrated by the tearing of a sheet of paper. In order to characterize crack propagation in torn thin films, we built a controlled setup enabling the study of both the stability of a single crack path and the interaction between two propagating cracks. Moreover, this experimental setup allowed the description of the focusing of the bending elastic energy from large scales to the crack tip, leading to the creation of new surfaces. Note that the interaction between two cracks was investigated in the case of thin films adhering to a hard substrate $(17 ; 18)$ : when a stripe of the film made by initiating two notches is pulled from the substrate, two straight, non parallel cracks propagate and merge forming 
a triangular shape (18).

Here, we investigate the propagation of one or two cracks in thin sheets, with a large scale mode III loading. In section 2, we describe the experimental setup. We first focus on single cracks, reporting experimental results in sections 3 and a first interpretation in section 4 . Finally, in section 5, we consider the paths of two cracks propagating simultaneously.

\section{Experimental setup}

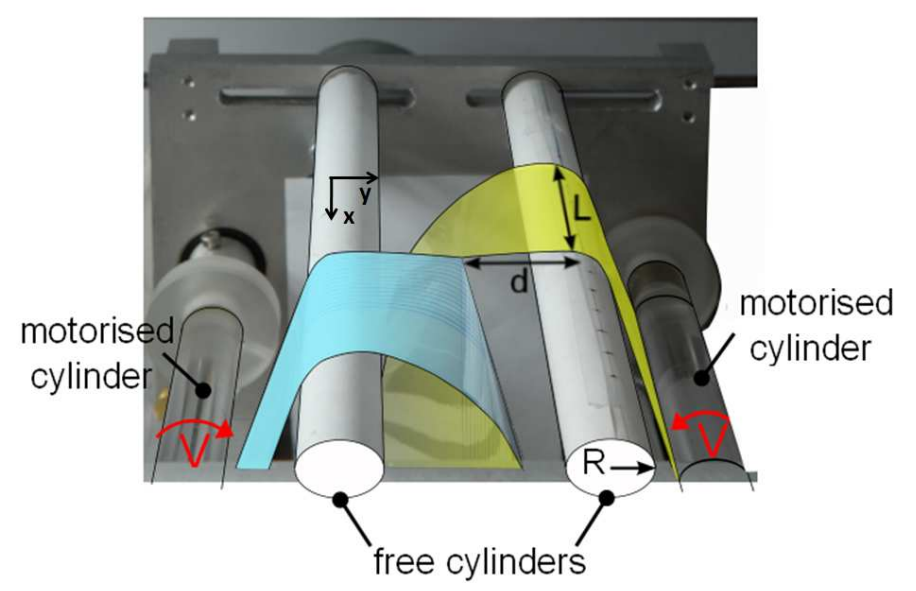

Fig. 1. The tearing machine was designed for an out-of-plane shear loading at a constant velocity. A thin film of width $2 L$, initially cut at its center, is introduced between the two upper cylinders, which are free to rotate. Here, one side of the cut (in front) is coloured in blue while the other (back) is coloured in yellow. Each stripe of the torn film is fixed, throughout its width, to one of the two lower counter-rotating cylinders, which are driven at the same constant velocity. The crack tip is located between the free cylinders, roughly at the same height as the top of cylinders. The distance between the axes of the two upper cylinders is $2 d$.

Inspired by the classical trousers test (13), we built an apparatus to control crack propagation at constant velocity in a thin elastic sheet submitted to an out-of-plane shear loading, mimicking the common way one tears a sheet of paper. The experimental setup consists of four parallel Plexiglas cylinders, of radius $8 \mathrm{~mm}$ and length $20 \mathrm{~cm}$, set between two parallel metallic plates (Fig. 1). The two upper cylinders are free to rotate, while the two lower cylinders are driven at the same constant velocity by a motor, so that the velocity at the surface of the cylinders is in the range $0.05-1.5 \mathrm{~mm} . \mathrm{s}^{-1}$. The elastic films are made of polypropylene and have isotropic mechanical properties. In order to study the propagation of a single crack, we initiate a straight centered cut in a long rectangular sample that we insert between the free cylinders. Each stripe of the torn sheet is fixed on each driven cylinder after passing above a free rotating cylinder. The distance between the two upper cylinders imposes the 
crack free length. The lower cylinders collect the torn parts of the sheet. The crack tip is generally located midway between the upper cylinders (Fig. 1). In order to study the propagation of two cracks, we use the same procedure: we start with two cuts that are symmetric with respect to the central axis of the sheet, determining three parts in the sheet; the two external parts are fixed on one of the driven cylinders while the middle part is fixed on the opposite cylinder.

The experimental setup allows us to control the following parameters: the distance $2 d$ between the two upper cylinders ( $d$ being the length of the free part of the crack), the crack propagation velocity, the sample width $2 L$ and the thickness $h$ of the sheet. We use films of bidirectional polypropylene of thickness 15, 30, 50 and $90 \mu \mathrm{m}$ and Young's modulus 2.2 $\pm 0.4 G P a$. At ambient temperature and in the range of the imposed tearing velocities, the fracture process is brittle for this material. We varied the width of the film from 2 to $16 \mathrm{~cm}$ and the crack free length $d$ from 1.8 to $5 \mathrm{~cm}$. This experiment requires a careful adjustment of the parallelism of the cylinders' axes in order to keep a symmetric loading.

The critical fracture toughness $K_{c}$ of polypropylene films was measured for cracks submitted to pure tensile loading. A constant displacement was applied on the edges of a rectangular sample of dimensions $22 \mathrm{~cm} \times 10 \mathrm{~cm} \times 30 \mu \mathrm{m}$ in which we initiated a centered notch of length $l$. We measure the value of the applied force when the crack starts to propagate. Initial values of crack length $l$ range from 0.4 to $6 \mathrm{~cm}$. Using the approximation of the infinite plane for the stress intensity factor, as given by $K=\sigma \sqrt{\pi l / 2}$, where $\sigma$ is the magnitude of the far-field stress, and applying the Irwin criterion we obtained $K_{c}=$ $2.6 \pm 0.3 M P a . m^{\frac{1}{2}}$ for the fracture toughness.

\section{$3 \quad$ Single crack propagation experiments}

\subsection{Stability analysis}

In a first experiment, we studied the stability of a single propagating crack. We found that within the present configuration the crack always follows a straight path parallel to the central axis of the sample even when the crack is initiated off the center of the sheet. In order to study the effect on the crack path when external perturbation are applied, we used a film of width $8 \mathrm{~cm}$ with an initially centered straight notch, in which we made three incisions of $1.4 \mathrm{~cm}$ at an angle of $45^{\circ}$ with respect to the direction of the initial notch (Fig. 2). The distance between two successive incisions, which have been made to perturb the crack path, was $5 \mathrm{~cm}$. In this configuration, the crack propagation starts by follow- 
ing a straight path. When the crack tip meets an incision, the configuration becomes asymmetric; nevertheless the crack keeps following an off-centered straight path that is parallel to the central axis of the sample (Fig. 2). Therefore, the crack path remains stable with respect to any perturbations. This makes the behaviour of the crack propagation in the tearing configuration fundamentally different from the case of in-plane propagation under opening mode for which the crack path can be either straight, propagating on the axis of the sample, or unstable $(3 ; 12)$.

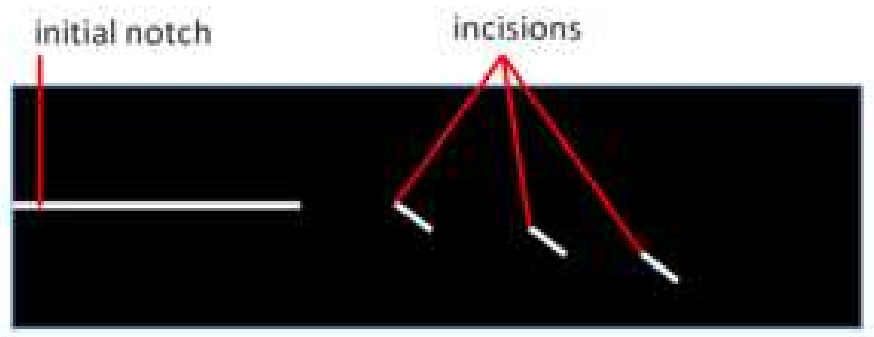

(a)

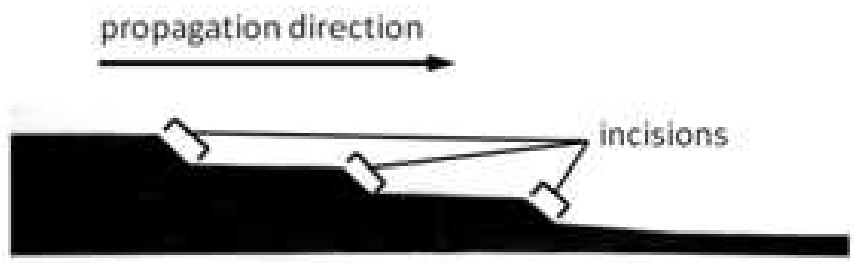

(b)

Fig. 2. Stability of the crack path. (a) Schematic of the film before tearing: we initiate a centered cut in the film and prepare inclined incisions in order to perturb the crack path. (b) Scan of the film after tearing: the crack path remains straight in the presence of perturbations along is path, even when it is off-centered or close to the boundary of the sample.

\subsection{Geometry of the tearing film}

In a second set of experiments, we considered the three-dimensional geometry of the film during the tearing process: three regions can be distinguished (see Figs. 1 and 3). A first region, far from the crack, is bent under the action of gravity and does not seem to be influenced by the presence of the crack (region 1 in Fig. 3). A second region hangs under the apparatus and is tilted by an angle $\theta$ with respect to the cylinders axes. As shown in Fig. 4(a), this angle is constant $\left(\simeq 18^{\circ}\right)$, independently of $L / d$, and thus, of the geometry of the experiment. The third region which is located in the neighborhood of the crack tip (region 3 in Fig. 3) has a shape close to a triangle with a vertex at the crack tip. The contour of this region delimits the area where the film is flat. The flatness of the film in this region can be interpreted as a sign that 
it is stretched and then contains most of the elastic energy that is used to open the crack. Notice that the geometry of the region 2 is mainly governed by the actual tearing process. The torn part is purely stretched near the crack surfaces (region 3) and it is purely bended far from it (region 1); the vertical part of the sheet being free to adapt its shape, must be tilted by angle $\theta$ in order suppress the excess of curvature at the boundaries of the sheet.

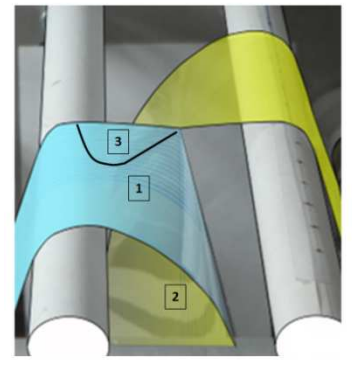

(a)

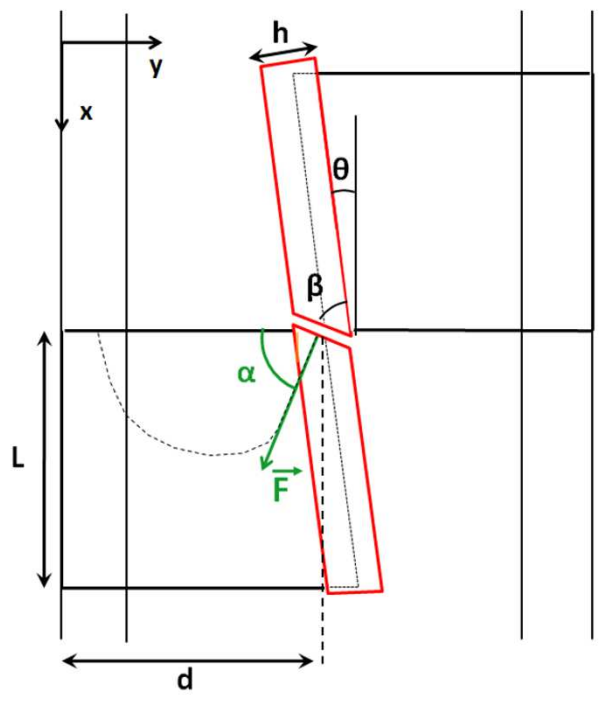

(b)

Fig. 3. (a) Zoom of Fig. 1. Region 1, which is located far from the crack surface, is bent due to gravity. Region 2 is located hangs vertically under the apparatus and is tilted by an angle $\theta$ with respect to the cylinders axes. Region 3 is a flat region whose a shape is close to a triangle ending at the crack tip. (b) Schematic of a top view of the experimental setup: the red rectangle shows a horizontal cut across the hanging vertical part of the film, with a magnified thickness. The opening angle of region 3 at the crack tip is $\alpha$, the bevel angle across the thickness of the torn film is $\beta$ while $\theta$ is the angle between the hanging part and the axes of the cylinders.

In order to investigate the geometry of the stretched part in Fig. 3, we performed strain measurements of the deformed elastic sheet. Unlike the experimental technique used in (16), we performed direct image analysis of the three-dimensional shape of the film as explained hereafter. First, we note that the curvature in the $x$-direction is very small in comparison with the curvature in the $y$-direction, so that we can neglect it. In order to visualize the curvature of the film, we draw on it parallel lines in the $y$-direction, equidistant by $2 \mathrm{~mm}$ : during crack propagation these lines will be straight in the stretched region only. We used degree-2 polynomial fits to measure the curvatures of these lines. We find that the flat region is bounded by the crack line and a straight line emanating from the crack tip, yielding a characteristic angle $\alpha$ (see Fig. 3). We measured $\alpha$ for an extensive range of experimental parameters (Fig. 4(b)) and found an approximately constant value $\alpha=58^{\circ} \pm 4^{\circ}$ that is insensitive neither to the aspect ratio $L / d$ nor to the film thickness $h$. Moreover, Fig. 4(b) 
shows that this angle is not correlated to the geometrical angle $\tan ^{-1}(L / d)$, thus the geometry of the stretched region is insensitive to the experimental setup and $\alpha$ characterizes the local concentration of stresses in the vicinity of the crack tip.
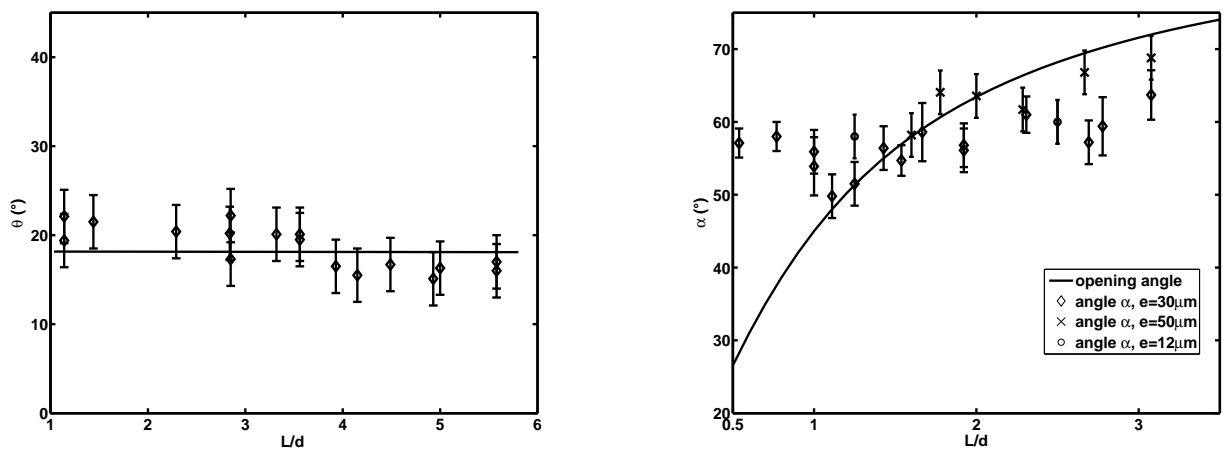

Fig. 4. (a) The angle $\theta$ as a function $L / d$ for a film of thickness $30 \mu m$. (b) The angle $\alpha$ of the "triangular" flat region as a function $L / d$ for three value of the film thickness. The opening angle is given by $\tan ^{-1}(L / d)$ and is defined by the crack line and the line joining the crack tip to the contact point of the film edge with the top cylinder.

As sketched in Fig. 3, in addition to the geometrical angles $\alpha$ and $\theta$, there exists a third angle that characterizes the tearing process across the thickness of the film. Indeed, post-mortem analysis of the crack shows a bevel in the thickness of the sample, which is absent when the sheet is torn under tensile loading (see Fig. 5(a)). Then this angle is a characteristic of the mode III loading induced by the present tearing process. Using a top view of the crack surface, we can deduce the bevel angle $\beta$ from the thickness of the film and the apparent width of the crack surface. Fig. 5(b) shows that for bidirectional polypropylene $\beta=45^{\circ} \pm 3^{\circ}$ independently of the experimental parameters $L / d$ and of the film thickness. Therefore, the latter three angles satisfy the identity $\alpha \simeq \beta+\theta$.

\section{Interpretation of the experimental results}

The curve limiting the flat region marks the transition from a region of zero curvature from a bent region of finite curvature. Therefore, this separation curve induces a line of maximum tension in the sheet which would correspond to a large scale pulling force on the crack tip. The fact that the different angles satisfy the condition $\alpha=\beta+\theta$, means that this line of maximum tension is perpendicular to the crack surfaces (Fig. 3). Consequently, even for thin sheets the system adapts itself geometrically so that the crack propagates under the action of a local pure tensile loading (mode I), although a large scale out-of- 

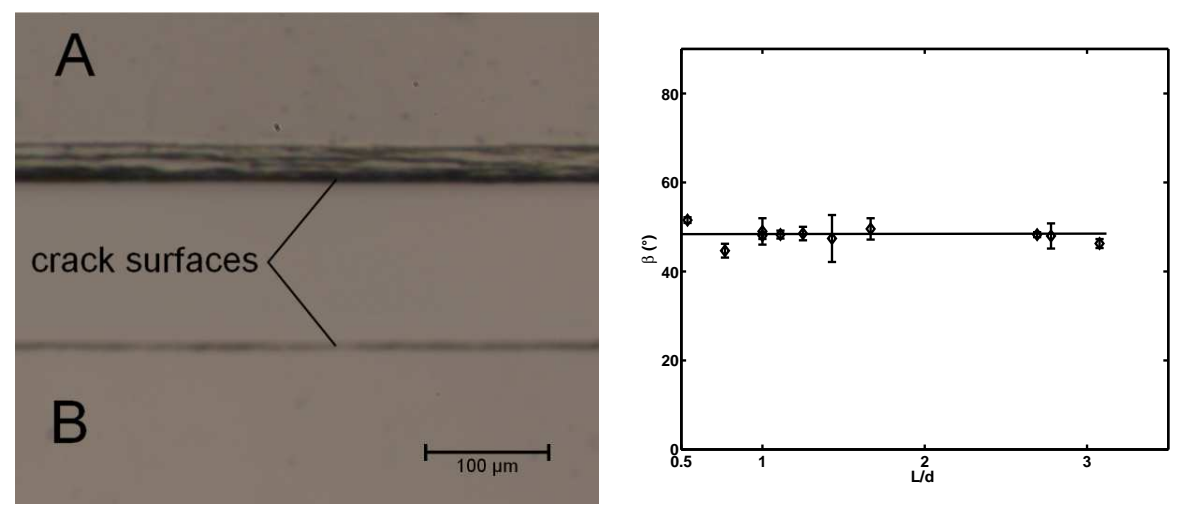

Fig. 5. (a) Magnified view with a microscope of the crack surface. A: a crack propagated in out-of-plane shear mode using the the present tearing apparatus. The crack surface is darker because it is rough. The width of the dark region shows that the crack surface is tilted, defining a bevel across the thickness of the film. $B$ : a crack propagated under tensile loading. The crack surface looks sharper, showing that it is normal to the surface of the film. (b) Bevel angle $\beta$ of the crack surface, as a function of the parameter $L / d$ for films of thickness $30 \mu \mathrm{m}$.

plane shear mode is imposed. Our result can be seen as a generalization of the Principle of Local Symmetry for a crack propagating in a thin plate teared under mode III loading at large scales.

Notice that the stress field induced by the crack tip is divergent and cannot be simply described by a force. The force we introduced above should be understood as the resultant of the stress field far from the tip. In order to validate our interpretation, we built a method to measure the intensity of this large scale force. We performed experiments where we increased the mass of the film by hanging weights at the free edge of the sample below the crack tip. The masses ranged from 1.45 to $7 \mathrm{~g}$ (the film mass is $0.8 \mathrm{~g}$ ). A first observation is that the angles $\alpha, \beta$ and $\theta$ are not affected by the mass of the film. However, the crack tip departs from the plane determined by the top of the free cylinders (see Fig. 6). We measured the height of the crack tip $z$ as a function of the applied mass $m$ (Fig. 6). As the bevel angle does not vary, the area of the new surfaces is not affected by the weights, so that the energy needed to create new surfaces remains constant. As a consequence, we may assume that the intensity of the force is independent of the experimental geometrical parameters. The balance of vertical forces is simply given by

$$
2 F_{0} \cos \alpha \sin \delta=m g
$$

with $\tan \delta=z / d$. In the experiments performed we had $z / d$. Then

$$
z=\frac{g d}{2 F_{0} \cos \alpha} m
$$


Indeed, Fig. 6 shows we that $z$ varies linearly with $d$ in the experimental range $0.1 \leq z / d \leq 0.3$. We could not investigate a wider range of masses because when $m$ is increased further a stick-slip-like phenomenon occurs, such that crack propagation is no more quasi-static. The force intensity is obtained with a linear fit as $F_{0}=0.25 \pm 0.05 \mathrm{~N}$. Then we compare this value with the minimal force necessary to propagate a crack in this material, as given by the critical toughness $K_{c}$. In the experiment, we observed that a force is applied in the horizontal plane far from crack tip. The typical distance $l$ between the effective application point of this force and the crack tip is of the order of the thickness. The necessary force to propagate the crack is given by $F_{t h}=K_{c} h \sqrt{\frac{2 l}{\pi}}$. For the $30 \mu \mathrm{m}$ thickness films, we obtain $F_{t h}=0.3 N$ which coincides, within the experimental uncertainty, with the measured value $0.25 \mathrm{~N}$. Thus the present experimental approach can be viewed as a new tool to measure the fracture toughness of thin sheets submitted to out of plane loading (a modified version of the trouser's test experiments).

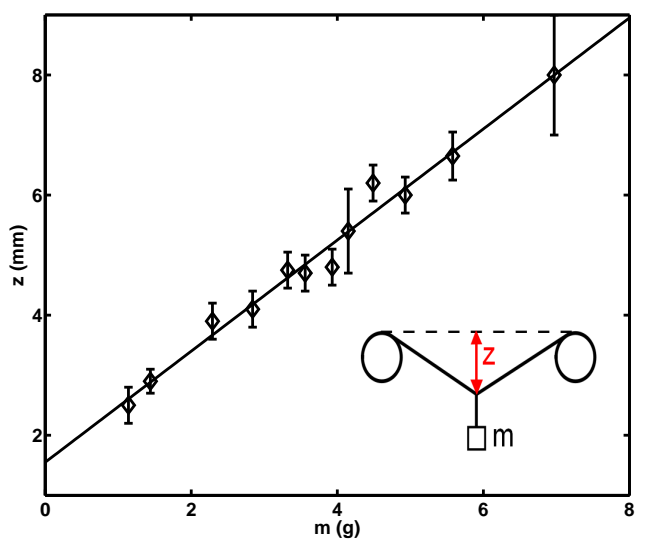

Fig. 6. The height of the crack tip $z$ as a function of the added mass $m$ at the bottom of the film, for a film of thickness $h=30 \mu \mathrm{m}, d=2.6 \mathrm{~cm}$ and $L=4 \mathrm{~cm}$.

\section{Cracks interaction experiments}

The interaction between two cracks was previously investigated in the case of thin films adhering to a hard substrate $(17 ; 18)$. Following these previous studies, we performed experiments to investigate the simultaneous propagation of two cracks using our experimental setup. We initiate two cracks symmetrically with respect to the central axis of the sample. The main control parameters of this experiment are the initial distance between the two cracks $l$ and the width of the sample $L$. We checked that the crack free length $d$ does not influence our results. In this new configuration, the two cracks trajectories are curved toward the central axis. Then after the merging of the cracks, the central piece of the sample detaches itself from the rest. The torn part of the sheet has a 


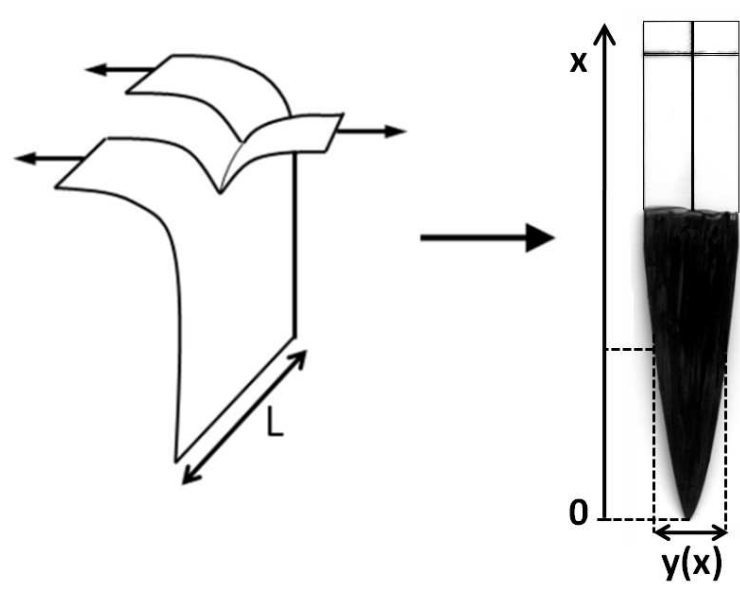

Fig. 7. Schematic of the experiment (left) and a scan of the resulting pattern from the simultaneous propagation of two cracks (right).

symmetric tongue-like shape (Fig. 7); When the ratio of the initial width of the central part with the width of the sample is small enough $(l \leq 0.1 L)$, experiments are reproducible. If the middle part is larger $(l \geq 0.1 L)$, experiments are less reproducible and the shape of the tear is not always symmetric. This could be due to the high sensitivity of the two-cracks propagation experiment to the imperfections in the parallelism of the cylinders' axes.

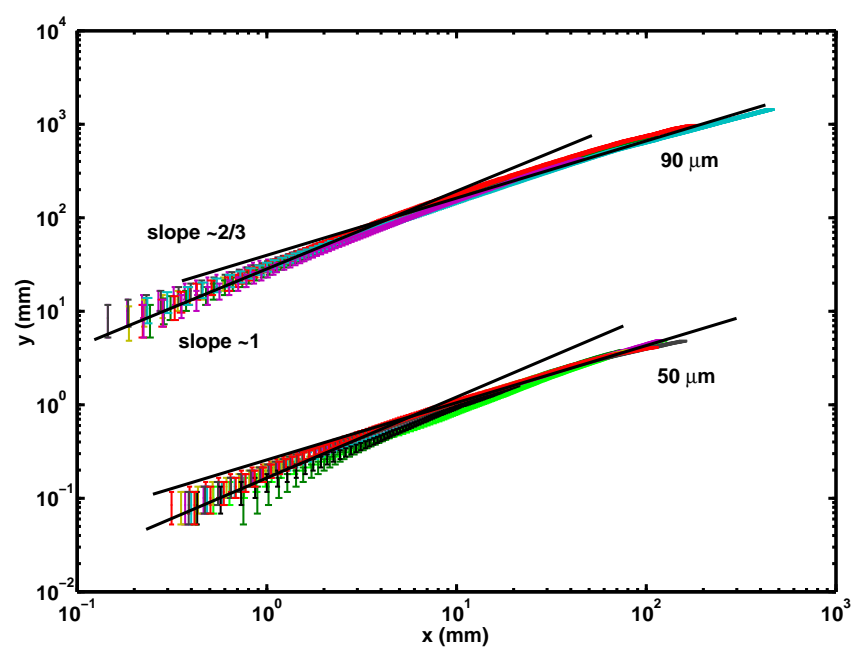

Fig. 8. Log-Log plots of the width of the strip along the direction of tearing. The merging point of the two cracks defines the origin of $x$-axis. The points corresponding to films of thickness $90 \mu \mathrm{m}$ are multiplied by $10^{2}$ for clarity. Experiments with the same film thickness and different distances $l$ between the two initial cuts clearly superimpose.

We digitized the tongue-like shapes and measured their width as function of the distance to their tips. Fig. 8 is the main result of this section. It shows that for a given thickness of the film, profiles can be superimposed for different values of the initial width $l$ and are well-described by power laws. Moreover, the 
prefactors of the power laws increase with the thickness, while the exponents appear to remain constant. Fig. 8 also shows that there is transition in the power law behavior when the width of the samples becomes small; i.e. close to the tip of the strip. The exponent at small scales is close to 1 and far from the tip, the value of the exponent is approximately 0.6. The transition occurs when the width of the sample is around $5 \mathrm{~mm}$. The change in the behavior of the two cracks paths when approaching their merging point might be related to the following observations on the resulting tear:

(1) During the tearing process, the cracks tips positions do not remain stationary and are not always located at the mid-distance $d$ between the two cylinders because the distance between the cracks tips is decreasing. This leads to an asymmetry in the loading configuration which in turn increases the bending deformation of the central part of the sheet.

(2) After tearing, far from the tip, the strip recovers its flatness indicating a brittle fracture process, while it is permanently curved in a small region close to the tip which is a signature of plastic deformations.

(3) Post-mortem analysis of the fracture surface across the film thickness shows that the bevel angle is far from the tip is constant along the strip and equal to $45^{\circ}$, in agreement with a single crack propagation. However, at approximately $3.5 \mathrm{~mm}$ from the cracks merging position, a transition occurs; the bevel angle suddenly increases until reaching the value of $63^{\circ}$ at the tip of the strip. When the width becomes of the order of the thickness, problem becomes intrinsically 3D.

These observations indicate that there are two regimes of fracture process. When the distance between cracks is large, the imposed loading for tearing is low leading to a brittle fracture behavior. In this regime, the strip is described by a power law shape with a well-defined exponent of approximately 0.6. A transition occurs when the distance between the crack tips is of order of few thicknesses of the film. In this region, the fracture process in no more brittle because of the high loading imposed by bending deformations. In this plastic regime, the strip becomes sharper and is well-described by a triangular shape. During the first regime of propagation when the distance between the two cracks tips is large, the bevel angle of both crack surfaces is the same as for a single crack propagation. This suggests that the local geometry at the crack tip, constrained by the necessity to break under mode I loading, is not modified.

\section{Conclusion}

The main result of the present experimental study is that crack propagation in a thin film submitted to out-of-plane tearing mode at large scale occurs such 
that the crack tip is opened locally under the action of an in-plane tensile loading. In other words, the fracture process is always ruled by the Griffith criterion and the Principal of Local Symmetry, whatever is the loading mode at large scales and the dimensionality of the material. Moreover, we have shown that, within our experimental setup, a single crack always propagate such that its path is always straight and parallel to the lateral boundaries of the sample and that it remains stable in the presence of imposed perturbations on its path. The present work can be put in the framework of the more general problem of stability of crack paths under mixed mode loadings.

Concerning the interaction of two cracks, we have shown that in the tearing mode two cracks propagating simultaneously attract each other and merge to form a tear of reproducible shape. Image analysis show that the obtained pattern is well described by a power law function with an exponent of order $2 / 3$ in the brittle fracture regime. The interpretation of this behavior will be the subject of a future study.

\section{Acknowledgements}

We are grateful to Laurent Quartier for his help in building the experimental apparatus and to Benoit Roman for insightful discussions.

\section{References}

[1] Freund LB. Dynamic Fracture Mechanics. Cambridge: Cambridge University Press; 1980.

[2] Leblond JB. Mécanique de la rupture fragile et ductile. Paris: Hermes Science Publications; 2003.

[3] Cotterell B, Rice JR. Slightly curved or kinked cracks. Int J Fracture 1980;16:155-169.

[4] Adda-Bedia M, Arias R, Ben Amar M, Lund F. Generalized Griffith criterion for dynamic fracture and the stability of crack motion at high velocities. Phys Rev E 1999;60:2366-2376.

[5] Griffith AA. The Phenomenon of Rupture and Flow in Solid. Phil Trans R Soc A (London) 1920;221:163-198.

[6] Gol'dstein RV, Salganik RL. Brittle fracture of solids with arbitrary cracks. Int J Fracture 1974;10:507-523.

[7] Leblond JB. Crack paths in plane situations- I. General form of the expansion of the stress instensity factors. Int J Solids Struct 1989;25:1311-1325.

[8] Erdogan G, Sih GC. On the crack extension in plates under plane loading and transverse shear. Journal of Basic Engineering 1963;85:519-527. 
[9] Bilby BA, Cardew GE. The crack with a kinked tip. Int J Fracture 1975;11:708-712.

[10] Amestoy M, Leblond JB. Crack paths in plane situations-II. Detailed form of the expansion of the stress instensity factors. Int J Solids Struct 1992;29:465-501.

[11] Katzav E, Adda-Bedia M, Arias R. Theory of dynamic crack branching in brittle materials. Int J Fracture 2007;143:245-271.

[12] Corson F, Henry H, Katzav E, Adda-Bedia M. Thermal fracture as a framework for quasi-static crack propagation Int J Fracture 2009;58:1-14.

[13] Mai YM, Cotterell B. The essential work of fracture for tearing of ductile metals. Int J Fracture 1984;24:229-236.

[14] Lazarus V, Buchholz FG, Wiebesiek J. In: Institute of Fundamental Technological Research, Polish Academy of Science (2006).

[15] Barenblatt GI, Cherepanov G. On brittle cracks under longitudinal shear. PMM 1961;25:1110-1119.

[16] Yan JH, Sutton MA, Deng X, Wei Z, Zavattieri P. Mixed-mode crack growth in ductile thin-sheet materials under combined in-plane and out-ofplane loading. Int J Fracture 2009;160:169-188.

[17] Atkins AG. Opposite paths in the tearing of sheet materials. Endeavour 1994;18: 2-10.

[18] Hamm E, Reis PM, Leblanc M, Roman B, Cerda E. Tearing as a test for mechanical characterization of thin adhesive films. Nature Materials 2008;7:386-390. 